\title{
A case of primary lung cancer lesion demonstrated by F-18 FDG positron emission tomography/computed tomography (PET/CT) one year after the detection of metastatic brain tumor
}

\author{
YUICHI OZEKI ${ }^{1}$, YOSHIYUKI ABE ${ }^{2}$, HIDEYUKI KITA ${ }^{3}$, KATSUMI TAMURA $^{2}$, \\ IKUKO SAKATA $^{2}$, JIRO ISHIDA ${ }^{2}$ and KIKUO MACHIDA ${ }^{2}$ \\ ${ }^{1}$ Department of Thoracic Surgery, National Defense Medical College, Namiki 3-2; \\ ${ }^{2}$ Tokorozawa PET Diagnostic Imaging Clinic, Higashisumiyoshi 7-5; ${ }^{3}$ Department of Neurosurgery, \\ Tokorozawa Chuoh Hospital, Kitaakitu 753-2, Tokorozawa, Saitama, Japan
}

Received February 4, 2011; Accepted April 27, 2011

DOI: $10.3892 / \mathrm{ol} .2011 .318$

\begin{abstract}
Cancer of unknown primary origin (CUP) is an aggressive disease with a poor prognosis. Metastatic brain tumors occur in approximately $15 \%$ of all cancer patients. F-18 2'-deoxy-2fluoro-D-glucose (FDG) positron emission tomography (PET) combined with computed tomography (PET/CT) contributes to the evaluation of cancer staging, although the benefits of PET/CT for detection of CUP origins has yet to be determined. In this study, we present a 37-year-old man with a brain tumor detected by magnetic resonance imaging. Surgical biopsy indicated a metastatic undifferentiated carcinoma, while clinical examination and a CT scan did not detect any abnormalities, with the exception of brain metastases. PET/CT did not reveal abnormal FDG uptake. PET/CT revealed abnormal intense FDG uptake in a small nodular lesion in the right lung 1 year following the detection of brain metastasis, and no other abnormal FDG uptake was observed elsewhere in the body. Right upper lobectomy and dissection of mediastinal lymph nodes were performed. The pathological diagnosis was poorly differentiated adenocarcinoma, which was similar to the brain metastatic lesion, and there was no lymph node metastasis. This case revealed an extremely rare lung cancer with primary lesions demonstrated by PET/CT 1 year after the detection of brain metastasis. This case reveals that F-18 FDG PET/CT imaging of CUP origin is capable of positively impacting on the identification of small primary tumor foci.
\end{abstract}

Correspondence to: Dr Yoshiyuki Abe, Tokorozawa PET Diagnostic Imaging Clinic, Higashisumiyoshi 7-5, Tokorozawa, Saitama 359-1124, Japan

E-mail: abe@toko-pet.or.jp

Key words: PET/CT, metastatic brain tumor, lung cancer, unknown primary origin

\section{Introduction}

Cancer of unknown primary origin (CUP) is an aggressive disease with a poor prognosis (1). The most frequently detected primary carcinomas are those that are concealed in the lungs or pancreas (2). Metastatic brain tumor is the most common intracranial tumor, occurring in approximately $15 \%$ of all cancer patients (3). Additionally, in up to $10 \%$ of these patients, even after a time-consuming and costly workup, the primary tumor tissue remains unknown. The development of new modalities such as F-18 2'-deoxy-2fluoro-D-glucose (FDG) positron emission tomography combined with computed tomography (PET/CT) has contributed to the evaluation of various types of human cancer, and the benefits of PET/CT for cancer staging are well established. FDG PET/CT detects more metastatic sites than other modalities, and discloses the site of the primary tumor in $20-40 \%$ of cases (2), while the usefulness of PET alone for the detection of CUP origin is controversial (4). Pelosi et al observed that the primary tumor site was correctly identified by FDG-PET/CT in 24 out of 68 patients (35.3\%) (5). The above data strongly support the diagnostic contribution of whole-body FDG-PET/CT scan in the evaluation of patients with CUP syndrome and indicate its use in an early phase of the diagnostic passageway to optimize patient management.

In the present study, we report a case of a 37-year-old man with an extremely rare lung cancer with a primary lesion that had been revealed by PET/CT 1 year after the detection of brain metastasis. This case revealed that ${ }^{18} \mathrm{~F}-\mathrm{FDG}$ PET/CT imaging of CUP syndrome is capable of impacting positively on the identification of small primary tumor foci.

\section{Patient and methods}

This study was performed with the patient's informed consent and with the approval of the Ethics Committees of our respective institutes (National Defense Medical College, Tokorozawa PET Diagnostic Imaging Clinic and Tokorozawa Chuoh Hospital).

A 37-year-old man complaining of headache was admitted to the Tokorozawa Chuoh Hospital. No abnormal findings 

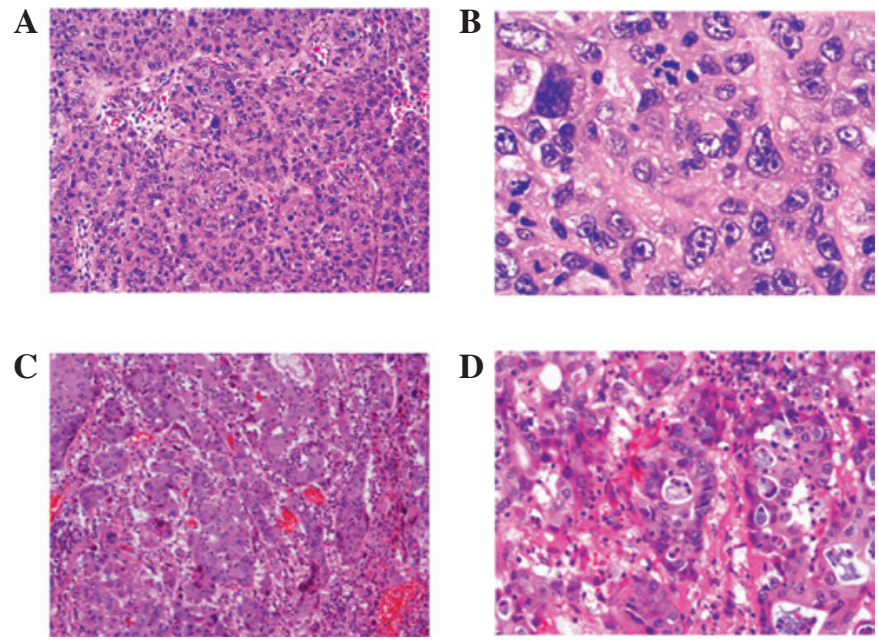

Figure 1. (A) Microscopic examination of brain tumor lesion showed foci of tumor growth of undifferentiated carcinoma, which suggested metastatic brain tumor. Magnification, x100. (B) Microscopic findings on high power view showed tumor cells with polygonal atypical nuclei. Magnification, x500. (C) Microscopic examination of right pulmonary tumor lesion showed foci of tumor growth of undifferentiated carcinoma, which was similar to that of a brain tumor lesion. Magnification, x100. (D) Microscopic findings of the right pulmonary tumor on high power view showed tumor cells with an abortive tubular formation, suggesting poorly differentiated adenocarcinoma. Magnification, x500.

were revealed by physical examination. Blood analysis did not reveal any remarkable abnormalities, including tumor markers such as carcinoembryonic antigen (CEA) and CA19-9. The patient had been a smoker ( $>30$ cigarettes/day) since the age of 15 years. Brain CT and magnetic resonance images revealed a brain tumor and surgery was performed. Biopsy specimens indicated metastatic undifferentiated carcinoma (Fig. 1A-B), while a systemic CT scan did not detect any abnormalities with the exception of a right pulmonary bulla (Fig. 2A).

The patient then underwent ${ }^{18} \mathrm{~F}-\mathrm{FDG}$ PET/CT scans. As described in our previous studies (6-8), ${ }^{18} \mathrm{~F}-\mathrm{FDG}$ PET/CT
A
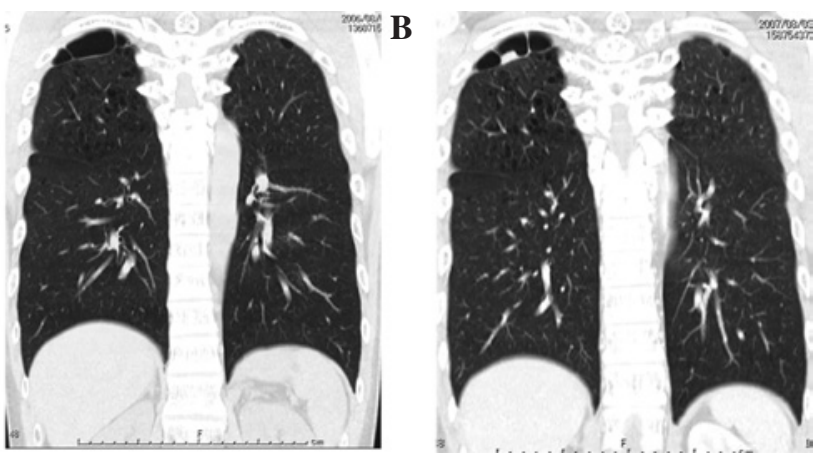

Figure 2. (A) Chest CT scan revealed pulmonary bulla in the apex of right lung at the time of surgery for the brain tumor. (B) Chest CT scan showed tumor lesion beside the pulmonary bulla 1 year after surgery on the brain tumor.

scans were obtained with a Biograph Duo (Siemens CTI) at Tokorozawa PET Diagnostic Imaging Clinic. To determine a semi-quantitative FDG uptake, regions of interest (ROIs) were placed over the lesion, including the highest uptake area (circular ROI, $1 \mathrm{~cm}$ in diameter), and the standardized uptake value (SUV) was calculated. PET/CT scan did not show any abnormal FDG uptake besides the non-specific uptake in the gastrointestinal tract (Fig. 3A). The patient was followed up in order to diagnose CUP origin without further therapy subsequent to resection of the brain tumor.

PET/CT showed abnormal intense FDG uptake (SUVmax: 5.09 in early scan, 5.22 in delayed scan) in the small nodular lesion beside the bulla in the right lung 1 year after resection of brain metastases. No other abnormal FDG uptake was observed elsewhere in the body (Fig. 3B-E). Right upper lobectomy and dissection of mediastinal lymph nodes were performed at the National Defense Medical College Hospital. The tumor in the right upper lobe measured $20 \mathrm{~mm}$ in diameter and was located beside the pulmonary bulla. The microscopic findings revealed the growth of atypical cells with abortive
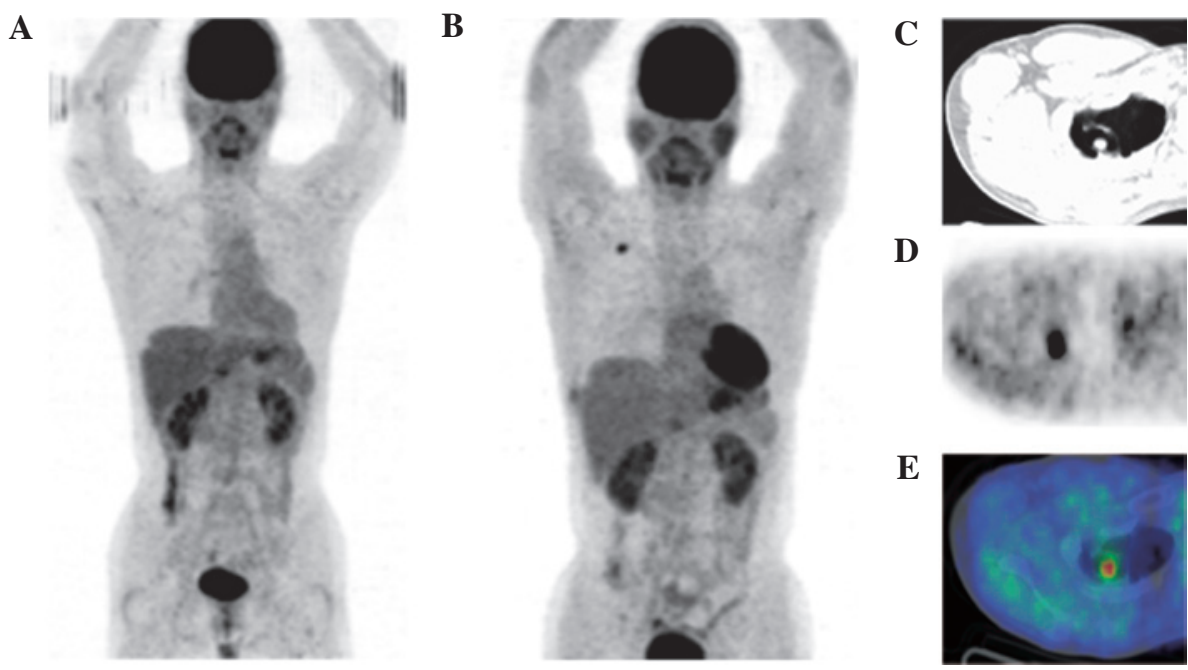

Figure 3. (A) Directly following surgery on the brain tumor, maximum intensity projection (MIP) PET imaging showed that there was no abnormal FDG uptake besides the non-specific uptake in the gastrointestinal tract. (B-E) MIP PET and PET/CT imaging showed abnormal intense FDG uptake in the tumor lesion beside the pulmonary bulla 1 year after surgery without other abnormal uptake, suggesting a primary lung cancer. 
glandular formation (Fig. 1C-D), and the pathological diagnosis was poorly differentiated adenocarcinoma, which was similar to the characteristics of the brain metastatic lesions. No metastatic lesions were observed in the dissected lymph nodes. PET/CT did not reveal any abnormalities 3.5 years after thoracotomy and there were no signs of either recurrence or systemic metastasis on any other examinations.

\section{Discussion}

Cancer of unknown primary (CUP) origin comprises a variety of different pathologic entities with a poor overall 5-year survival. Brain tumors are classified into primary and secondary tumors or metastases. Findings of a retrospective cohort study that included 63 adults with metastatic brain tumors showed lung cancer to be the most common source (9). Following definitive treatment of locally advanced non-small cell lung cancer (NSCLC), the risk of developing brain metastases is considered to be $30-50 \%$. In their study, Hubbs et al reported that in 975 NSCLC patients treated surgically during early stage I-II lung cancer, the 5-year actuarial risk of developing brain metastases was $10 \%$ (10). Furthermore, results of the multivariate analysis revealed that younger age, larger tumor size, lymphovascular space invasion and hilar lymph node involvement were correlated with an increased risk of developing brain metastases. Pimiento et al reported that the pathologic diagnoses of 91 CUP patients included adenocarcinoma (42.8\%), undifferentiated carcinoma (34.5\%), squamous cell carcinoma $(9.8 \%)$, neuroendocrine cancer $(6.5 \%)$, sarcoma (3.2\%), and non-specific malignant neoplasm (3.2\%) (1). Nonetheless, squamous cell and neuroendocrine CUP are associated with a significantly more favorable early prognosis than other malignancies. The patient in the present study remains alive with no recurrence for 4.5 years following brain tumor resection, while the pathology was poorly differentiated adenocarcinoma.

The development of FDG-PET/CT has contributed to the evaluation of human cancer staging and its benefits with regards to cancer staging have been well established. This imaging modality enables the otolaryngologist and radiation oncologist to treat head and neck cancer patients more effectively and leads to appropriate management changes (11). Pelosi et al reported that the primary tumor site was correctly identified by FDG-PET/CT in 24 out of 68 patients $(35.3 \%)$, including the lung $(n=9)$, rhino/oro-pharynx $(n=6)$, pancreas $(n=5)$, and other sites. (5). Salem et al reported that occult squamous cell carcinoma of the uvula was detected using ${ }^{18} \mathrm{~F}-\mathrm{FDG}$ PET/CT in a case of CUP in the head and neck (12). In this case, the first PET/CT scan did not reveal any abnormal FDG uptake, indicating a limitation of PET/CT with regard to the detection of extremely small foci.
In conclusion, we have reported for the first time a rare primary lung cancer lesion that was revealed by PET/CT 1 year following the detection of brain metastasis. Additionally, it was noted that F-18-FDG FDG PET/CT imaging of CUP origin is capable of impacting positively on the identification of small primary tumor foci.

\section{Acknowledgements}

We thank Mr. Kenji Kawai for technical assistance.

\section{References}

1. Pimiento JM, Teso D, Malkan A, Dudrick SJ and Palesty JA: Cancer of unknown primary origin: a decade of experience in a community-based hospital. Am J Surg 194: 833-838, 2007.

2. Pavlidis N, Briasoulis E, Hainsworth $J$ and Greco FA: Diagnostic and therapeutic management of cancer of an unknown primary. Eur J Cancer 39: 1990-2005, 2003.

3. Wu AH, Drees JC, Wang H, VandenBerg SR, Lal A, Henner WD and Pillai R: Gene expression profiles help identify the tissue of origin for metastatic brain cancers. Diagn Pathol 26: 26, 2010.

4. Podoloff DA: PET/CT and occult primary tumors. J Natl Compr Canc Netw 7: 239-244, 2009.

5. Pelosi E, Pennone M, Deandreis D, Douroukas A, Mancini M and Bisi G: Role of whole body positron emission tomography/ computed tomography scan with $18 \mathrm{~F}$-fluorodeoxyglucose in patients with biopsy proven tumor metastases from unknown primary site. Q J Nucl Med Mol Imaging 50: 15-22, 2006.

6. Ueda S, Tsuda H, Asakawa $H$, Shigekawa T, Fukatsu K, Kondo N, Yamamoto M, Hama Y, Tamura K, Ishida J, Abe Y and Mochizuki H: Clinicopathological and prognostic relevance of uptake level using 18F-fluorodeoxyglucose positron emission tomography computed tomography fusion imaging (18F-FDG PET/CT) in primary breast cancer. Jpn J Clin Oncol 38: 250-258, 2008.

7. Abe Y, Tamura K, Sakata, I, Ishida J, Mukai M, Ohtaki M, Nakamura $M$ and Machida K: Unique intense uptake demonstrated by $18 \mathrm{~F}-\mathrm{FDG}$ positron emission tomography/computed tomography (PET/CT) in primary pancreatic lymphoma: A case report. Oncol Lett 1: 605-607, 2010.

8. Abe Y, Tamura K, Sakata, I, Ishida J, Fukuba I, Matsuoka R, Shimizu S, Murakami $\mathrm{H}$ and Machida K: Usefulness of 18F-FDG positron emission tomography/computed tomography for diagnosis of pyothorax-associated lymphoma: A report of three cases. Oncol Lett 1: 833-836, 2010.

9. Popović N and Kalacun D: Origin and distribution of brain metastases. Med Pregl 57: 617-621, 2004.

10. Hubbs JL, Boyd JA, Hollis D, Chino JP, Saynak M and Kelsey CR: Factors associated with the development of brain metastases: analysis of 975 patients with early stage nonsmall cell lung cancer. Cancer 116: 5038-5046, 2010.

11. Fleming AJ Jr and Johansen ME: The clinician's expectations from the use of positron emission tomography/computed tomography scanning in untreated and treated head and neck cancer patients. Curr Opin Otolaryngol Head Neck Surg 16: 127-134, 2008.

12. Salem S, Patel NH, Barwick T, Al-Nahhas A, Howard DJ, Zerizer I and Win Z. Occult squamous cell carcinoma of the uvula detected by F-18 FDG PET/CT in a case of carcinoma of unknown primary in the head and neck. Clin Nucl Med 35: 800-801, 2010. 